\title{
An extension of a property of the phase space trajectories of a third order differential equation.
}

by J. O. C. EzerLo (Department of Mathematics, University of Ibadan, Tbadan, Nigeria)

Summary. - In the peesent paper it is shown that an earlier result [1], and its subsequent generalization [2] concerning the motion of trajectories in the phase space of the dif. ferential equation (11) can be extended to the more general equation (2.1), and this is done under a considerably weaker condition on $h(x)$.

1. Introduction. - It was proved in a previous note [1] dealing with the equation

$$
\dddot{x}+\alpha \ddot{x}+b \dot{x}+h(x)=p(t)
$$

in which $\alpha, b$ are positive constant that if (i) $h(0)=0, h(x) / x \geq \delta>0(x \neq 0)$, (ii) $h^{\prime}(\dot{x})$ is continuous and $\left|h^{\prime}(x)\right| \leq 0$ for all $x$ where $\alpha b>C^{\prime} / \delta$, (iii) $|p(t)| \leq$ $\leq A_{0}<\infty$ for all $t$ considered, then there exists a bounded surface $\Sigma$ in the phase space, $E_{3}$, of (1.1) such that every directed line from the origin meets $\Sigma$ in only one point and which has the property that trajectories in $E_{3}$ cross $\Sigma$ only inwards. A generalization of this result was later given in [2], the object of which was to report that (i) and (ii) above are unduly restrictive on $h(x)$ and may indeed be replaced by the weaker conditions: (i) $h(x) / x>0(|x| \geq 1)$ and $|h(x)| \rightarrow \infty$ as $|x| \rightarrow \infty$, (ii) $h^{\prime}(x) \leq \gamma<\alpha b(|x| \geq 1)$ and a constant $\Gamma>0, \infty b>\Gamma \geq \gamma$, exists such that

$$
\left.\left.\max _{|x| \leq 1} \| \Phi^{\prime}(x)\right]^{2}-4 \mathrm{~T} \Phi(x)\right\}<+\infty
$$

where $\Phi \equiv x h(x)$; but, for lack of space, no indications of the proof were given in [2].In the present note we shall see that the restriction (1.2) can be further relaxed without affecting the result in [2], but our main object is to extend the result to the more general case in which the term $p$ depends on $x, \dot{x}, \ddot{x}$ as well as on $t$. Either of the earlier results in [1], [2] or their present generalization, which is stated in $\S 2$, may be regarded as a three dimensional analogue of Levinson's result [4; Theorem II].

2. Statement of the result. - We consider then slightly more general equation

$$
\dddot{x}+\alpha \ddot{x}+b \dot{x}+h(x)=p(t, x, \dot{x}, \ddot{x})
$$


388 J. O. C. Ezmo: An extension of a property of the phase space, etc.

in which $p$ depends on all the arguments shown. It will be assumed as basic throughout what follows that $h$ and $p$ are such that solutions of (2.1) exist satisfying any assigned initial conditions. Also we shall retain the notation $E_{s}$ for the space of all solutions $(x, y, z)$ of the equivalent system:

$$
\dot{x}=y, \dot{y}=z, \dot{z}=-\alpha z-b y-h(x)+p(t, x, y, z)
$$

obtained from (2.1) on setting $y=\dot{x}$ and $z=\dot{y}$.

The following result will be proved:

Theorem. Suppose that

$$
\text { (i) } \alpha>0, b>0, h(x) / x>0 \quad(|x| \geq 1) \text {, }
$$

(ii) $|h(x)| \rightarrow \infty$ as: $x \mid \rightarrow \infty$,

(iii) $h^{\prime}(x)$ is continuous for all $x$ and $h^{\prime}(x) \leq \gamma<\alpha b(|x| \geq 1)$,

(iv) a constant $\mathrm{\Gamma}>0, a b>\Gamma \geq \gamma$, exists

such that

$$
\max \left(0,\left[\left|\Phi^{\prime}(x)\right|^{2}-4 \Gamma \Phi(x)\right]\right)=0\left(x^{8}\right)
$$

for $|x| \geq 1$, where $\Phi(x) \equiv x h(x)$ and $\varepsilon$ is a constant such that $0 \leq \varepsilon<2$,

(v) the function $\theta(R)$ defined, for any $R_{\mathrm{u}} \geq 0$, by

$$
\theta(R)=\max _{t \geq 0,: x|\leq R, y| \leq R,|z| \leq R}|p(t, x, y, z)|
$$

satisfies

$$
\theta(R)=o(h( \pm R))
$$

as $R \rightarrow+\infty$.

Then there is a bounded surface $\Sigma$ in $E_{3}$ such that every directed line issuing from the origin meets $\Sigma$ in one point only and which has the properties :

(I) all trajectories of (2.2) cross $\Sigma$ only inwards,

(II) any trajectory of (2.2) starting outside $\Sigma$ ultimately enters $\Sigma$.

Observe that, subject to the condition (ii), a function $p(t, x, y, z)$ satisfying (v) above may well be unbounded for arbitrarily large $x^{2}+y^{2}+z^{2}$.

In general the conditions (iii) and (iv) are quite independent but there are certain rather special cases of (2.1) for which (iii) is implied by (iv). For example consider the case where $\Phi$ is such that

$$
\left[\Phi^{\prime}(x)\right]^{2}-4 \Delta \Phi(x) \leq 0
$$


J. O. C. EzeiLo: An extension of a property of the phase space, etc. 389

for $|x| \geq 1, \Delta$ being a constant such that $0<\Delta<a b$. Here, evidentty,

$$
\max \left(0,\left[\left|\Phi^{\prime}(x)\right|^{2}-4 \Delta \Phi(x)\right) \equiv 0 \quad(|x| \geq 1)\right.
$$

so that (2.3) is satisfied with $\mathrm{\Gamma}=\Delta$. On the other hand, if $\Phi \equiv x h(x)>0$ $(|x| \geq 1)$, one may obviously reset $(2.4)$ in the form

$$
\begin{aligned}
& \Delta \geq\left(x h^{\prime}+h\right)^{2} /(4 x h) \\
& =\frac{1}{4}\left\{2 h^{\prime}+\frac{h}{x}+\frac{h^{\prime 2}}{h / x}\right\} .
\end{aligned}
$$

But, by the inequality between the geometric and arithmetic mean,

$$
\frac{h}{x}+\frac{h^{\prime 2}}{h / x_{j}} \geq 2 \mid h^{\prime}
$$

Hènce, subject to an assumption that $h / x>0$ for $|x| \geq 1$, (2.4) im* plies that

$$
\frac{1}{2}\left(h^{\prime}+\left|h^{\prime}\right|\right) \leq \Delta
$$

and this in turn implies that

$$
h^{\prime}(x) \leq \Delta
$$

which is hypothesis (iii) of the theorem.

3. Notation. - In what follows $C_{1}>0, C_{2}>0^{-}$are constants such that

$$
h^{\prime}(x) \leq C_{1} \text { and }|h(x)| \leq C_{2} \quad(|x| \leq 1)
$$

and $\delta_{1}>0, \delta_{2}>0$ are fixed constants chosen (as is possible since $a b>\Gamma>0$ ) such that

$$
\alpha^{-1}<\delta_{1}<b / \Gamma, \quad \alpha b-\Gamma>\alpha \delta_{2}>0 .
$$

The symbols $D, D_{0}, D_{1}, D_{2}, \ldots$, wherever they oocur in the sequel, denote finite positive constants whose magnitudes depend only on $\alpha, b, C_{1}, C_{2}, \delta_{1}, \delta_{2}$, $\Gamma$ and, sometimes, on how $|h(x)| \rightarrow \infty$ as $|x| \rightarrow \infty$ or on how $|\theta(R) h( \pm R)| \rightarrow 0$ as $R \rightarrow \infty$ in hypothesis (v). These $D^{\prime} s$ are not necessarily the same 
390 J. O. C. EzemL : An extension of a property of the phase space, etc.

in each place unless a suffix is attached. In lemma 3 , we allow ourselves the use of a "free» constant $k, 0<k<\infty$, whose value is chosen later on (in the application of lemma (3) as a $D$.

4. Some lemmas. - The methods of the present note are inspired by certain properties (stated here as lemmas 1,2 and 3) of the function $V=V(x$, $y, z)$ introduced in [3] and which, in terms of the notation of the present note, may be defined by

$$
V=V_{1}+V_{2}-\frac{8 C_{1}}{\pi}\left(1+\delta_{1}\right) y \times(x)
$$

where $\chi$ is the differentiable function of $x$ :

$$
\chi=\left\{\begin{array}{cc}
1 & , x>2, \\
\sin (\pi x / 4, & , x \leq 2, \\
-1, & x<-2,
\end{array}\right.
$$

and $\nabla_{1}, V_{2}$ are given by

$$
\left\{\begin{array}{c}
2 V_{1}=2 \int_{0}^{x} h(\xi) d \xi+\left(\alpha+\delta_{1} b\right) y^{2}+\delta_{1} z^{2}+2 y z+2 \delta_{1} y h(x) \\
2 V_{2}=\delta_{2} b x^{2}+2 \alpha \int_{0}^{x} h(\xi) d \xi+\left(\alpha^{2}+b-\delta_{2}\right) y^{2}+z^{2}+2 \alpha \delta_{2} x y+ \\
+2 \delta_{2} x z+2 \alpha y z+2 y h(x)
\end{array}\right.
$$

$\delta_{1}$ and $\delta_{2}$ being the constants introduced earlier in $\S 3$.

Lemma 1. - Subject to the conditions (i), (ii) and (iii) of the theorem there exist constants $D_{0}, D_{1}$ and $D_{2}$ such that, for any solution $(x, y, z)=(x(t)$, $y(t), z(t))$ of $(2.2)$

$$
\begin{aligned}
\dot{V} \equiv \frac{d}{d t} V(x, y, z) & \leq-\left\{\delta_{2} x h(x)+D_{0}\left(y^{2}+z^{2}\right)\right\}+ \\
& +D_{1}(|x|+|y|+\mid z)|p(t, x, y, z)|+D_{2}|z|
\end{aligned}
$$

This result can be seen to follow at once from certain results obtained in the course of the proof of the second part of the lemma in $[3 ; \& 2]$. 
J. O. O. EzerLo: An extension of a property of the phase space, etc. 391

Further details of its proof are therefore omitted.

Lemma 2. - Subject to the conditions (i), (ii) and (iii) of the theorem, there are constants $D_{3} . D_{4}, D_{5}$ and $D_{6}$ such that

$$
D_{3}\left(x^{2}+y^{2}+z^{2}\right)-D_{4} \leq V(x, y, z) \leq D_{5}\left(x^{2}+y^{2}+z^{2}\right)+D_{6}
$$

for all $x, y$ and $\approx$.

Proof. - For the left hand side inequality we shall refer to an inequality :

$$
\begin{aligned}
2 V & \geq \delta_{2}\left(b-\delta_{2}\right) x^{2}+\left(\alpha-\delta_{1}^{-1}\right)+\left(b-\delta_{2}-\Gamma \alpha^{-1}\right)\left\{y^{2}+\left(z+\alpha y+\delta_{2} x\right)^{2}+\right. \\
& +\delta_{1}\left(z+\delta_{1}{ }^{-1} y\right)^{2}-2 D|y|-D \\
& =V_{0},
\end{aligned}
$$

say, which was obtained in $[3 ; \S 2]$ with $c$ in place of $\Gamma$ but under precisely the same conditions as in the present lemma. Since the terms $\delta_{1}, \delta_{2}$, $b-\delta_{2}, \alpha-\delta_{1}{ }^{-2}, b-\delta_{2}-\Gamma \alpha^{-2}$ are, by $(31)$. all positive, there exists a constant $D$ such that

$$
V_{0} \geq D\left(x^{2}+y^{2}+z^{2}\right)-D
$$

for all $x, y, z$ and the left half of $(4,5)$ follows at once.

We turn then to the right half of (4.5). Since the terms $x y, y z, z x$ occuring in (4.3) satisfy the inequalities

$$
|x y| \leq \frac{1}{2}\left(x^{2}+y^{2}\right), \quad y z\left|\leq \frac{1}{2}\left(y^{2}+z^{2}\right),\right| z x \mid \leq \frac{1}{2}\left(x^{2}+z^{2}\right)
$$

the right half of (4.5) will follow as soon as it is shown that

$$
|y \chi(x)| \leq D y^{2}+D,|y h(x)| \leq D\left(x^{2}+y^{2}\right)+D, \int_{0}^{\infty} h(\xi) d \xi \mid \leq D x^{2}+D
$$

for all $x, y$.

Concerning the term $y \chi(x)$ it is clear, since $|\chi(x)| \leq 1$ for all $x$, that

$$
|y \chi(x)| \leq|y| \leq y^{2}+1
$$


392 J. O. C. EzHILO: An extension of a property of the phase space, etc.

for all $x, y$ and this verifies the first inequality in (4.6) To deal with the other two we note that, since $h(x) \operatorname{sgn} x>0$ for $|x| \geq 1$, the fact that

$$
h(x) \mid \leq C_{1} \text { for }|x| \leq 1 \text { and } h^{\prime}(x) \leq \Gamma \text { for } \mid x_{i} \geq 1
$$

implies necessarily that

$$
|h(x)| \leq C_{1}+\Gamma|x|
$$

for all x. Hence

$$
|y h(x)| \leq C_{1}|y|+\Gamma|x y| \text { and }\left|\int_{0}^{x} h(\xi) d \xi\right| \leq C_{1}|x|+\frac{1}{2} \Gamma^{\prime} x^{2}
$$

for al $x, y$; and, since

$$
|y| \leq 1+y^{2},|x| \leq x^{2}+1,|x y| \leq \frac{1}{2}\left(x^{2}+y^{2}\right)
$$

for all $x$ and $y$, the second and third inequalities in (4.6) now follow. This completes the proof of lemma 2.

Lemma 3. - If the conditions (i), (ii) and (iii) of the theorem hold then for every $k, 0<k<\infty$, the surface $V \cdot x, y, z)=k$ is bounded. If, in addition $h$ satisfies the condition (iv) of the theorem then there exists $D_{7}$ such that every directed line issuing from the origin intersects the surface $V(x, y, z)=k$ in exactly one point, provided that $k \geq D_{7}$.

Proof. - The first part of the lemma follows readily from Lemma 2. In fact, let $(x, y, z)$ be any point of the surface $V(x, y, z)=k$ and assume that the conditions (i), (ii) and (iii) of the theorem hold. Then, by (4.5),

$$
x^{2}+y^{2}+z^{2} \leq\left(D_{4}+k\right) / D_{3}
$$

and thus all points of the surface $V(x, y, z)=k$ are bounded provided that $k$ is finite.

To deal with the second part of the lemma assume now that condition (i)-(iv) of the theorem are satisfied. Let

$$
\frac{x}{l}=\frac{y}{m}=\frac{z}{n}
$$

be any directed line issuing from the origin, where $l, m, n$ satisfy

$$
l^{2}+m^{2}+n^{2}=1 \text {. }
$$


J. O. C. EzemL: An extension of a property of the phase space, etc. 393

It is clearly enough to prove that, for all $l, m, n$ satisfying (4.7) there is one and only one real $r, 0<r<\omega$, satisfying the equation

$$
\psi(r) \equiv V(l r, m r, n r)-k=0
$$

for finite $k \geq D$.

Now, from the definition $((4.1),(4.2)$ and $(4.3))$ of $V$, we have that

$$
\psi(0)=-k<0 \text {. }
$$

Also, by (4.5) and (4.7),

$$
\begin{aligned}
\psi(r) & >D_{3} r^{2}-D_{4}-k \\
& >0
\end{aligned}
$$

for sufficienty large $r$. Since $\psi(r)$ is continuous in $r$ the two results about $\psi(0)$ and $\psi(r)$ imply that there is at least one real root of (4.8). If $k$ is sufficiently large there cannot be more than one root. For suppose that, for some $l, m, n$ and for a certain $k>0$ there were two roots $r_{1}, r_{2}\left(r_{2} \geq r_{1}>0\right)$ of (4.8), so that

$$
\psi\left(r_{1}\right)=\psi\left(r_{2}\right)=0
$$

Then, by Rolle's Theorem, we shall have that

$$
\psi^{\prime}(R)=0
$$

for some $R, 0<r_{1} \leq R \leq r_{2}$. By (4.1) and and (4.3) $\psi^{\prime}(R)$ has the explicit form

$$
\psi^{\prime}(R)=\psi_{1}(R)+\psi_{2}(R)-\frac{8 C_{1}}{\pi}\left(1+\delta_{1}\right)\left\{m \chi(l R)+l m R \chi_{\chi}^{\prime}(l R)\right\}
$$

where

$$
\begin{gathered}
\psi_{1} \equiv\left(\alpha+\delta_{1} b\right) m^{2}+\delta_{1} n^{2}+2 m n ! R+l h(l R)+\delta_{1} m h(l R)+\delta_{1} l m R h^{\prime}(l R), \\
\psi_{2} \equiv\left(\delta_{2} b l^{2}+\left(\alpha^{2}+b-\delta_{2}\right) m^{2}+n^{2}+2 \alpha \delta_{2} l m+2 \alpha m n+2 \delta_{2} n l l R+\right. \\
+\alpha l h(l R)+m h(l R)+l m R h^{\prime}(l R) .
\end{gathered}
$$

Verify from these definitions that

$$
\begin{gathered}
\psi_{1}=R\left\{\delta_{1}\left(n+m / \delta_{1}\right)^{2}+\left(\alpha-\delta_{1}{ }^{-1}\right) m^{2}\right\}+\frac{1}{R}\left\{\delta_{1} b\left[m R+\frac{\Phi^{\prime}(\lambda)}{2 b}\right]^{2}\right\}+ \\
+\frac{1}{R}\left\{\Phi(\lambda)-\frac{\delta_{1}}{4 b}\left[\Phi^{\prime}(\lambda)\right]^{2}\right\}
\end{gathered}
$$


394 J. O. C. Ezemo: An extension of a property of the phase space, etc.

$$
\begin{array}{r}
\left.\left.\psi_{2}=R\left|\delta_{2}\left(b-\delta_{2}\right) l^{2}+\right| b-\delta_{2}-\Gamma / \alpha\right) m^{2}+\left(\delta_{2} l+\alpha m+n\right)^{2}\right\}+ \\
\left.+\frac{1}{\bar{R} \mid \Gamma} \mid \frac{\alpha}{\alpha}\left[m R+\frac{\alpha}{2 \Gamma} \Phi^{\prime}(\lambda)\right]^{2}+\alpha\left[\Phi(\lambda)-\frac{1}{4 \Gamma}\left(\Phi^{\prime}(\lambda)\right)^{2}\right]\right\}
\end{array}
$$

where

$$
\lambda \equiv l R \text { and } \Phi(\lambda)=\lambda h(\lambda)
$$

Since $\delta_{2}, b-\delta_{2}, b-\delta_{2}-T / \alpha$ are, by (3.1), all positive, it is clear that the term

$$
\delta_{2}\left(b-\delta_{2}\right) l^{2}+\left(b-\delta_{2}-\Gamma / \alpha\right) m^{2}+\left(\delta_{2} l+\alpha m+u\right)^{2}
$$

in (4.12) is a positive definite quadratic form in $l, m, n$. Thus, since $l, m, n$ satisfy (4.7), there exists $D_{8}$ such that

$$
\delta_{2}\left(b-\delta_{2}\right) l^{2}+\left(b-\delta_{2}-\Gamma / \alpha\right) m^{2}+\left(\delta_{2} l+\alpha m+n\right)^{2} \geq D_{9} .
$$

Also note that the term

$$
\Phi(\lambda)-\delta_{1}\left[\Phi^{\prime}(\lambda)\right]^{2} / 4 b
$$

in (4.11) may be rewritten in the form

$$
\begin{aligned}
\Phi(\lambda)-\frac{\delta_{1}}{4 b}\left[\Phi^{\prime}(\lambda)\right]^{2} & =\Phi(\lambda)-\frac{1}{4 \Gamma}\left[\Phi^{\prime}(\lambda)\right]^{2}+\frac{1}{4}\left\{\frac{1}{\Gamma}-\frac{\delta_{1}}{b}\right\}\left[\Phi^{\prime}(\lambda)\right]^{2} \\
& \geq \Phi(\lambda)-\left[\Phi^{\prime}(\lambda)\right]^{2} / 4 \Gamma,
\end{aligned}
$$

since $\delta_{1} / b<1 / \Gamma$, by $(3.1)$. Hence, on adding $\phi_{1}$ to $\phi_{2}$, we have that there is a constant $D_{9}$ such that

$$
\psi_{1}(R)+\psi_{2}(R) \geq D_{8} R-\frac{D_{9}}{R}\left[\left[\Phi^{\prime}(\lambda)\right]^{2}-4 \Gamma \Phi(\lambda)\right\}
$$

where $\lambda, \Phi$ are defined by (4.13). Concerning the thind term on the right hand side of (4.10) it is clear from the definition (4.2) that $|\chi(x)| \leq 1$ for all $x$ and that $\left|\chi^{\prime}(x)\right|=0$ for $|x|>2$. Thus, since (4.7) implies, in particular, that $|m| \leq 1$, we have that

$$
\left|m \chi(l R)+l m R \chi_{\chi}^{\prime}(l R)\right| \leq D
$$

for all $l, m, R$. Hence, putting all these results into (4.10), we have that

$$
\psi^{\prime}(R) \geq D_{8} R-\frac{D_{9}}{R}\left\{\left[\Phi^{\prime}(\lambda)\right]^{2}-4 \mathrm{\Gamma} \Phi(\lambda)\right\}-D .
$$


J. O. C. EzEILO: An extension of a property of the phase space, etc. 395

Thus, since $|\lambda| \equiv|l R| \leq R$, it follows, on using (2.3) that there is a constant $D_{10}$ such that

$$
\psi^{\prime}(R)>0
$$

provided that $R \geq D_{10}$. However, the constant $R$ of particular interest to us here satisfies $r_{1} \leq R \leq r_{2}$, where $r_{1}>0$ is a root of the equation

$$
V(l r, m r, n r)=k \text {. }
$$

By $(4, \overline{0})$ one can make this root $r_{1}$ larger than $D_{10}$ merely by taking $k$ large enough; in fact, since $l^{2}+m^{2}+n^{2}=1$, the root $r_{1}$ necessarily satisfies

$$
D_{5} r_{1}^{2} \geq k-D_{6}
$$

by (4.5), so that $r_{1} \geq D_{10}$ is achieved if $k$ is chosen such that

$$
k \geq D_{6}+D^{2}{ }_{10} D_{5} \equiv D_{11}
$$

In other words, if $k \geq D_{11}$ then $R \geq r_{1} \geq D_{10}$ and so $\psi^{\prime}(R)$ satisfies (4.14). As (4.14) itself is a contradiction to (4.9) it follows then that the equation (4.8) cannot have more than one root if $k \geq D_{11}$. This proves the lemma completely.

5. Proof of the theorem. - To establish the theorem we shall show that the surface $S(k)$ defined by $V(x, y, z)=k$ has all the properties stated in the conclusion of the theorem if $k$ is large enough.

By lemma 3, if $k \geq D_{\tau}$ the surface $S(k)$ is bounded and is met in exactly one point by any directed line. issuing from the origin. It thus remains to verify that the properties (I) and (II) stated in the theorem are also fulfilled on $S(k)$ for $k$ sufficiently large. For this it suffices to show that, if $(x(t), y(t)$, $z(t))$ is any solution of $(2.2)$ then

$$
\dot{V} \equiv \frac{d}{d t} V(x(t), y(t), \approx(t)) \leq-D<0
$$

at every point where the trajectory $\left(x(t), y^{\prime}(t), z(t)\right)$ meets the surface $S(k)$, provided that $k \geq D$.

The proof of (5.1) depends on the estimate of $\dot{V}$ given in Lemma 1. Let $\zeta=\zeta(t)$ be defined by

$$
\zeta=\max (|x(t)|,|y(t)|,|z(t)|)
$$


396 J. O. C. EzwiLo: An extension of a property of the phase space, ete.

and recall here the definition of $\theta(R)$ in hypothesis (v) of the theorem. Suppose first that $\xi=|x|$. Then, by (4.4),

$$
\dot{V} \leq-\delta_{2} x h(x)+3 D_{1}|x| \theta(|x|)+D_{2}|x|, \quad(t \geq 0) .
$$

Since $h(x) / x>0(|x| \geq 1)$, the terms on the right hand side of the ine. quality $(5.2)$ may be written in the form

$$
-|x|\left|\delta_{2}\right| h(x)\left|-3 D_{1} \theta(|x|)-D_{2}\right|
$$

provided that $|x| \geq \mid$. By hypotheses (ii) and (v) of the theorem there exists $D_{12} \geq 1$ such that

$$
\delta_{2} \mid h\left(x \mid-3 D_{1} \theta(|x|)-D_{2} \geq 1 \text { for }|x| \geq D_{12} .\right.
$$

Hence, when $\zeta=|x|$,

$$
\dot{V} \leq-D_{12}
$$

provided that $|x| \geq D_{12}$. Next suppose that $\zeta=|y|$. Then, since $h(x)_{i} x>0$ for $|x| \geq 1$ and $|h(x)| \leq C_{2}$ for $|x| \leq 1$, we see here, from (4.4), that, for all $t \geq 0$,

$$
\dot{V} \leq-D_{0} y^{2}+3 D_{1}|y| \theta(|y|)+D_{2}|y|+\delta_{2} C_{2} \text {. }
$$

In order to be able to ascertain more precisely the sign of the expression of the right hand side for large $|y|$ we shall first show that,

$$
\theta(R)=o(R) \text { as } R \rightarrow+\infty \text {. }
$$

Indeed let $R \geq 1$. Then, by hypothesis (i) of the theorem, $h(R) \neq 0$, and it is then valid to express the ratio $\theta(R) / R$ in the form

$$
\frac{\theta(R)}{R}=\frac{\theta(R)}{|h(R)|} \cdot \frac{|h(R)|}{R}
$$

But, as has already been shown in the course of the proof of lemma 2,

$$
|h(R)| \leq C_{1}+\Gamma R
$$

Thus

$$
\begin{aligned}
\frac{\theta(R)}{R} & \leq \frac{\theta(R)}{h(R) \mid} \cdot \frac{C_{1}+\Gamma R}{R} \\
& \rightarrow 0
\end{aligned}
$$


J. O. C. Ezmilo: An extension of a property of the phase space, ete. 397

as $R \rightarrow \infty$, since $\theta(R)=o(h(R))$. To return now to the inequality (5.3) we note that, if $y \neq 0$, the inequality may be put in the form:

$$
\dot{V} \leq-|y|\left\{D_{0}|y|-3 D_{1} \theta(|y|)-D_{2}-\delta_{2} C_{2} /|y| 1 .\right.
$$

In view of (5.4) it is obvious that there is a constant $D_{13}$ such that

$$
D_{0}|y|-3 D_{1} \theta(|y|)-D_{2}-\delta_{2} C_{2} /|y| \geq 1 \text { for }|y| \geq D_{13} \text {. }
$$

Hence, when $\zeta=|y|, \quad \dot{V}$ satisfies

$$
\dot{V} \leq-D_{13}
$$

provided that $|y| \geq D_{13}$. In the same way if $\zeta=|z|$ we shall have that

$$
\dot{V} \leq-D_{13}
$$

provided that $|z| \geq D_{13}$. Hence, on combining all three results, we have that

$$
\dot{V} \leq-\min \left(D_{12}, D_{13}\right)
$$

if $x^{2}+y^{2}+z^{2} \geq D_{14} \equiv 3\left\{\max \left(D_{12}, D_{13}\right)\right\}^{2}$. But, by (4.5), points $(x, y, z)$ of the surface $V(x, y, z)=k$ satisfy $x^{2}+y^{2}+z^{2} \geq D_{14}$ if

$$
k \geq\left(D_{\mathrm{G}}+D_{5} D_{14}\right) \equiv D_{15} \text {. }
$$

Hence, if $k \geq D_{15}$ then

$$
\dot{V} \leq-D<0
$$

and every point where the trajectory $(x(t), y(t), z(t))$ meets the surface $S k k)$. The surface

$$
V(x, y, z)=\max \left(D_{7}, D_{15}\right)
$$

thus has all the properties ascribed to $\Sigma$ in the theorem; and the verification of the theorem is now complete:

\section{REFERENCES}

[1] J. O. C. Ezello, J. London Math. Soc. " 37 (1962), 33.11.

[2] J. O. C. Ezano, Int. Congress of Mathematicians: abstracts of short comunications Stockholm (1962), 74-75.

[3] J. O. C. Ezmilo, J. London Math. Soc. 38 (1963), 11.16.

[4] N. Levinson, «J. Math. Phys. 22 (1943), 41-48. 